\title{
Reflets
}

Revue ontaroise d'intervention sociale et communautaire

\section{Les mémoires, essais et thèses de maîtrise en service social}

Céline Ayotte, Diane Boisjoli, Monette Boudreau-Roth, Louise Mulligan-Roy, Donna Quesnel, Hélène Simard, Patricia Smith, Sharon Stern et Linda Sullivan

Volume 3, numéro 1, printemps 1997

Enfance et familles en contexte d'appauvrissement

URI : https://id.erudit.org/iderudit/026165ar

DOI : https://doi.org/10.7202/026165ar

Aller au sommaire du numéro

Éditeur(s)

Reflets : Revue ontaroise d'intervention sociale et communautaire

ISSN

1203-4576 (imprimé)

1712-8498 (numérique)

Découvrir la revue

Citer ce document

Ayotte, C., Boisjoli, D., Boudreau-Roth, M., Mulligan-Roy, L., Quesnel, D., Simard, H., Smith, P., Stern, S. \& Sullivan, L. (1997). Les mémoires, essais et thèses de maîtrise en service social. Reflets, 3(1), 176-186.

https://doi.org/10.7202/026165ar

Tous droits réservés (C) Reflets : Revue ontaroise d'intervention sociale et communautaire, 1997
Ce document est protégé par la loi sur le droit d'auteur. L'utilisation des services d'Érudit (y compris la reproduction) est assujettie à sa politique d'utilisation que vous pouvez consulter en ligne.

https://apropos.erudit.org/fr/usagers/politique-dutilisation/ 


\title{
La perception de jeunes face à leur démarche de changement par rapport à un problème de surconsommation de drogues : «Changer un mode de vie»
}

\author{
Céline Ayotte \\ École de service social, Université d'Ottawa
}

Ce mémoire de maitrise porte sur la perception de certains jeunes face à leur démarche de changement grâce à une intervention en matière de toxicomanie. La surconsommation de drogues chez les jeunes est un phénomène relativement récent et surtout considéré comme un problème social dans les pays industrialisés. Ce phénomène s'inscrit dans un contexte social complexe. En effet, la société véhicule des messages contradictoires face à certains types de drogues d'une part, et face à des modèles de consommation d'autre part. Plusieurs cadres d'analyse se sont attardés à tenter d'expliquer les causes de cette surconsommation chez les jeunes. Parmi ceux-ci, l'approche écologique a le mérite de percevoir l'individu en interaction avec différents systèmes d'influence. Sa richesse réside donc dans la vision globale qu'elle nous offre de la problématique. Par ailleurs, différents modèles et modalités d'intervention ont tenté d'aider ces jeunes à résoudre ce problème. À l'égard de ceux-ci, un débat émerge: c'est la confrontation entre les tenants d'un modèle d'intervention dont la finalité est l'abstinence et un autre où l'emphase est mise sur la réduction de la consommation.

Dans cette étude exploratoire de type qualitatif, nous avons tenté de mieux comprendre le cheminement de trois (3) adolescentes et de trois (3) adolescents francophones à OttawaCarleton ayant entrepris une démarche de changement face à 
leur surconsommation de drogues. L'objet de la recherche est de saisir la perception de ces jeunes face à leur démarche. Pour ce faire, nous mettons en lumière leur processus de réflexion rétrospectif suite à une intervention en milieu externe, soit le programme de jour de La Maison Fraternité pour adolescentes et adolescents. Au cours de leur cheminement, ces jeunes ont eu la possibilité d'opter pour l'abstinence ou pour la réduction de la consommation comme objectif principal.

Cette recherche se veut donc un outil de réflexion en ce qui a trait à la problématique des jeunes ayant un problème de surconsommation de drogues. À partir d'une perspective d'analyse écologique, nous apportons une attention particulière à la famille, aux pairs et au milieu scolaire en tant que facteurs importants d'appuis au cheminement ou d'obstacles à ce dernier.

\section{Les femmes survivant à la violence conjugale: leurs difficultés et leurs stratégies pour se construire une vie sans violence}

\section{Diane Boisjoli}

École de service social, Université d'Ottawa

La problématique de ce mémoire explore les liens entre la pauvreté, la violence conjugale et les difficultés de logement.

Après avoir identifié les diverses formes de violence dont sont victimes les femmes, cette étude se penche sur la situation du logement au Canada, et plus précisément celle du logement social en Ontario. Des liens sont alors établis entre la dépendance économique des femmes et les difficultés financières qui surviennent quand elles quittent des conjoints violents, notamment celle de se trouver un logement permanent et abordable.

Les entrevues auprès de six femmes survivantes de violence conjugale ont révélé de quelle façon elles se heurtent aux valeurs 
sociales qui préconisent le maintien de l'unité nucléaire familiale. Elles souffrent d'avoir un revenu insuffisant, un fait qui a des répercussions sur tous les aspects de leur vie. Elles n'arrivent pas à se loger convenablement sur le marché privé ou à subvenir aux autres besoins de leur famille. Leurs relations avec les propriétaires sont pour la plupart difficiles, et les logements sociaux ne sont pas toujours la réponse.

Les séquelles de leur vécu de violence continuent donc à se faire sentir dans leur vie. Elles sont socialement marginalisées parce qu'elles sont pauvres, sans conjoint, survivantes de violence conjugale, femmes et mères monoparentales.

Malgré cela, ces femmes cherchent à jouer un rôle valable dans la société. Elles attribuent beaucoup d'importance à leur rôle de mères. Elles désirent réintégrer le marché du travail et sont prêtes à entreprendre la formation nécessaire pour le faire. Elles contribuent à leur communauté par du travail bénévole, par des échanges de services ou en devenant entrepreneuses.

\title{
Les complexités des pratiques interculturelles dans le social - une approche souple et en évolution
}

\begin{abstract}
Monette Boudreau-Roth
École de service social, Université d'Ottawa

La présente étude s'est penchée sur la lecture que font les intervenantes et les intervenants sociaux de leur relation d'aide auprès des personnes immigrantes, des personnes réfugiées et des demandeurs d'asiles politiques.

Comment les services sociaux peuvent-ils se mettre au diapason des changements démographiques des populations qui composent notre société actuelle? Comment les intervenantes et les intervenants sociaux, dans leur rôle auprès de personnes d'autres cultures dans le besoin peuvent-ils devenir des chefs de file et établir des programmes d'appoint pour répondre aux attentes
\end{abstract}


légitimes des nouvelles et des nouveaux arrivants? Enfin, comment s'acquitter de sa tâche pour que la personne immigrante, la personne réfugiée ou le demandeur d'asile politique puisse passer de l'infime comparse à l'acteur qui va sur le pied de guerre pour s'adapter à sa nouvelle situation de vie.

Le travail est complexe puisque nous avons parfois le sentiment que le «système» se laisse distancer par les changements qui se produisent dans notre société. Les pratiques interculturelles s'avèrent de plus en plus importantes et nécessitent une plus grande compréhension du vécu pré et post-migratoire des individus qui viennent d'ailleurs. Il ne faut pas battre en retraite face à ces défis mais accepter de questionner nos valeurs à celles d'une population plus diversifiée. Dans un monde qui ne cesse d'évoluer, nous pouvons puiser une très grande richesse dans les différentes cultures avec lesquelles nous transigeons en tant qu'intervenantes et intervenants sociaux.

\section{Autonome, malgré tout: stratégies d'autonomie chez des aînées franco-ontariennes ayant reçu un diagnostic de perte d'autonomie}

\section{Louise Mulligan-Roy}

École de service social, Université d'Ottawa

Le concept d'autonomie peut être vu comme le droit d'une personne à déterminer librement les règles auxquelles elle se soumet. Ce concept renvoie à ceux de liberté et d'indépendance. Pourtant, présentement, la notion d'autonomie dans le contexte du vieillissement se réduit le plus souvent à la capacité de la personne âgée de fonctionner physiquement et psychologiquement et elle s'inscrit généralement dans un modèle déficitaire. Les perspectives fonctionnelle et psychologique de l'autonomie imposent, en fait, une définition des personnes âgées comme "problème». 
Une recadrage s'impose afin de saisir la réalité des personnes âgées en "perte d'autonomie» telle qu'elle est vécue quotidiennement par ces dernières. La perspective d' "autonomie fondamentale» que nous adoptons repose sur le respect des choix de l'aînée sur sa vie, les droits et les devoirs qui encadrent ces choix, et sur les ressources personnelles et collectives dont elle peut disposer pour les réaliser. Une interrogation a servi de guide à cette étude: quelles sont les stratégies et les frontières de l'autonomie dans la perspective des aînées franco-ontariennes recevant des soins, participant à un programme de jour et ayant été diagnostiquées en «perte d'autonomie»? Il ne s'agit pas d'énumérer le nombre de stratégies élaborées par les aînées ou les frontières, mais plutôt de saisir la forme qu'elles prennent et le sens que les aînées leur accordent. Une méthode de recherche exploratoire de type qualitatif, axée sur des entrevues semi-dirigées auprès d'aînées franco-ontariennes ayant reçu un diagnostic de "perte d'autonomie», a été privilégiée afin de rendre compte de la complexité de leur réalité et de la richesse de leurs discours.

Une analyse descriptive des discours des aînées nous permet de cerner leurs caractéristiques socio-démographiques, les difficultés et les enjeux auxquels elles sont confrontées dans leur quotidien ainsi que la nature des liens qu'elles entretiennent avec leur entourage. Une analyse interprétative de ces mêmes discours font apparaître les aînées comme des actrices qui élaborent des stratégies de contournement, de dégagement et de compensation afin d'exercer leur autonomie fondamentale. Pour y arriver, les stratégies d'autonomie mises en oeuvre par les aînées s'appuient surtout sur leurs réseaux sociaux, et plus précisément la famille et les amitiés.

Ces constats nous amènent à une réflexion sur les implications de ces connaissances pour l'intervention et la recherche auprès des aînées franco-ontariennes ayant des difficultés de santé. 


\section{"C'est difficile, mais je ne le regrette pas» Etre mère et adolescente francophone en Ontario: détermination de situations problèmes et de stratégies d'existence}

\section{Donna Quesnel}

École de service social, Université d'Ottawa

La maternité à l'adolescence demeure une préoccupation importante, car de plus en plus de jeunes femmes choisissent de garder et d'élever leur enfant. Cependant, le vécu des mèresadolescentes d'aujourd'hui differe considérablement de celui des «filles-mères» de jadis; la question de la maternité à l'adolescence est loin d'être statique. Ces mères ne forment plus un groupe homogène, et de multiples scénarios sont maintenant possibles. Bien que les nombreux écrits publiés à ce jour dressent un portrait plutôt sombre de la situation des jeunes mères, peu de recherches ont essayé de comprendre le sens de cette expérience pour la principale concernée.

La présente étude consiste donc en une recherche de type qualitatif explorant la signification de la maternité à l'adolescence pour les jeunes mères. La collecte de données s'est effectuée par le biais d'entrevues semi-dirigées menées auprès de huit mèresadolescentes franco-ontariennes qui sont inscrites à un programme pour parents-adolescents offert dans une école alternative.

L'exploration du vécu des mères-adolescentes quant aux situations problèmes rencontrées et aux stratégies d'existence mises en oeuvre nous mène à une réflexion sur les implications de leurs propos au regard des politiques sociales et des pratiques suivies en matière d'intervention. 


\section{«Il y a moins de sexualité... mais il y a plus d'amour»: La sexualité des personnes ayant un vécu psychiatrique}

\section{Hélène Simard}

École de service social, Université d'Ottawa

Cette recherche traite du vécu des personnes psychiatrisées dans une activité de la vie humaine: la sexualité. Nous avons tenté d'explorer le sens qu'elles donnent à l'expression de leur sexualité dans une société où leur différence les écarte du circuit des rapports sociaux.

Dans le cadre de cette démarche, nous avons effectué des entrevues individuelles, semi-dirigées, auprès de six personnes ayant un vécu psychiatrique et demeurant dans la communauté. Nous visions à démontrer que la stigmatisation attachée à leur condition les contraignait dans le développement et l'épanouissement de leur sexualité. L'exclusion et la marginalisation dont elles sont victimes se transposent dans la quête de l'autre et leur vécu amoureux. Il se dégage des données recueillies que leur sexualité est multidimensionnelle, qu'elle comporte des aspects affectifs, relationnels et sociaux et qu'elle ne peut être dissociée de leur vécu.

Trois intervenants et intervenantes ont aussi été interviewés afin d'obtenir leur perception de la sexualité des personnes ayant un vécu psychiatrique. L'analyse du discours dans les pratiques d'intervention qui mettent en cause la sexualité des personnes psychiatrisées suscite des réflexions et des questionnements. On note l'existence d'interdits et le peu de place accordées à l'expression de la sexualité dans le mouvement d'intégration qui a marqué l'après-désinstitutionnalisation.

Ce constat nous amène à changer nos conceptions traditionnelles à l'égard du vécu psychiatrique des personnes dans 
l'expression de leur sexualité et à replacer dans le contexte historique, social et culturel toute la question de la folie et de la sexualité.

\section{La violence en milieu scolaire et une méthode pour la contrer : perceptions des jeunes et du corps professoral}

Patricia Smith

École de service social, Université d'Ottawa

Ce mémoire rend compte d'une recherche exploratoire concernant la violence en milieu scolaire et les méthodes d'intervention pour la prévenir. Ses objectifs sont de présenter et d'analyser les perceptions du personnel et des élèves par rapport aux causes et solutions de la violence et d'explorer certaines pistes de solutions. Les études actuelles ne s'accordent ni sur l'ampleur ni sur les causes ou solutions de la violence en milieu scolaire. Parmi les interventions préventives recensées, on retrouve les solutions de type tertiaire telle la politique de tolérance zéro, les programmes de prévention secondaire telle la médiation par les pairs et enfin, les interventions visant la promotion ou la prévention primaire qui cherchent à améliorer le climat ou à augmenter les compétences.

Nous avons réalisé notre étude par l'entremise d'une observation participante de 4 mois dans une école secondaire francophone qui accueille une importante population multiculturelle. L'enquête s'est faite au moyen d'observations informelles et formelles auprès des élèves de la $9^{\mathrm{e}}$ à la $12^{\mathrm{e}}$ année, de même qu'auprès de 11 membres du personnel, le tout suivi par une analyse de contenu. Les résultats attestent des diverses formes que peut prendre la violence ainsi que de son ampleur. Cinq causes ressortent:le racisme, l'influence des pairs, les problèmes individuels 
et familiaux, le climat et l'attitude au sein de l'école. Cependant, l'importance attribuée à l'une ou l'autre des causes varie selon les acteurs. Les pistes de solution proposées comprennent des actions directes cherchant spécifiquement à contrer la violence et les actions indirectes visant à améliorer l'environnement scolaire. Cette étude conclut qu'une réponse adéquate au problème de la violence en milieu scolaire comporte nécessairement les deux types d'action. De plus, on se doit d'impliquer le personnel enseignant aussi bien que les élèves dans la recherche de solutions.

\title{
Étude exploratoire sur les facteurs de stress reliés au travail professionnel en milieu psychiatrique
}

\author{
Sharon Stern \\ École de service social, Université d'Ottawa
}

L'importance du travail pour l'être humain dans notre contexte moderne est étroitement relié au développement de l'identité et constitue une source de valorisation.Toutefois, le travail peut aussi être une source de stress et éventuellement mener à l'épuisement professionnel.

Afin de connaitre les principaux facteurs de stress reliés au travail professionnel, et les stratégies d'existence pour composer avec ceux-ci, nous avons entrepris une recherche de nature qualitative et exploratoire auprès de cinq travailleurs sociaux oeuvrant en milieu psychiatrique.

Une analyse qualitative du discours de ces travailleurs sociaux nous a fourni certaines réponses; ainsi ce sont les caractéristiques organisationnelles qui sont surtout associées aux facteurs de stress reliés au travail professionnel en milieu psychiatrique. On souligne aussi les changements organisationnels, la pression reliée au travail, l'absence de ressources et les conflits reliés au rôle. 
Quant aux stratégies d'existence, on remarque que les travailleurs sociaux favorisent la formation professionnelle, les relations humaines et un ensemble d'activités socio-professionnelles qui créent un équilibre entre le monde du travail et les besoins personnels. Finalement, nous proposons quelques pistes de solution, entre autres, les "groupes Balint», en tant que source de soutien professionnel.

\section{La pratique de maintien à domicile et les séjours raccourcis à l'hôpital: pour une autonomie de l'intervention dans la communauté}

Linda Sullivan

École de service social, Université d'Ottawa

Ce travail explore les nombreux contextes qui ont influencé et qui continuent d'influencer la formulation des politiques et des pratiques de maintien à domicile. Originalement conçu comme moyen de diminuer les coûts croissants liés à l'institutionnalisation des personnes âgées, le maintien à domicile a pris de l'ampleur en Ontario et au Canada et représente aujourd'hui une des politiques sociales les plus importantes en ce qui concerne les personnes âgées, en raison de leur haut niveau d'utilisation des services.

Les séjours raccourcis à l'hôpital, pratique plus récente que le maintien à domicile, ne constituent pas une politique sociale mais relèvent plutôt du contexte de rationalisation des budgets des hôpitaux et de la croyance que les gens guérissent plus rapidement dans un milieu familier que dans une institution de soins. Parce qu'elles utilisent un grand nombre de lits d'hôpitaux, les personnes âgées sont affectées par le raccourcissement des séjours à l'hôpital.

L'enquête présentée dans ce mémoire a pour objet d'explorer l'impact des séjours raccourcis à l'hôpital sur la pratique de 
maintien à domicile auprès des personnes âgées francophones d'Ottawa-Carleton.Afin d'explorer cet impact, nous avons recueilli les perceptions d'intervenantes dans la communauté. L'analyse de leurs propos indique que la pratique en situation de séjour raccourci à l'hôpital, tout en ayant une spécificité en ce qui concerne la pratique, présente des similitudes importantes avec la pratique quotidienne de maintien à domicile. Nous présentons donc une analyse de cette pratique en fonction du contexte et du contenu du travail tel que perçu par les intervenantes interrogées, ainsi que les enjeux sociaux qui se rapportent à l'impact des séjours raccourcis à l'hôpital sur la pratique du maintien à domicile. 